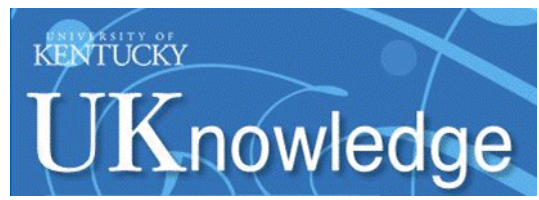

University of Kentucky

UKnowledge

Spinal Cord and Brain Injury Research Center Faculty Publications

\title{
Rod-Shaped Microglia Morphology Is Associated with Aging in 2 Human Autopsy Series
}

\author{
Adam D. Bachstetter \\ University of Kentucky, adam.bachstetter@uky.edu \\ Eseosa T. Ighodaro \\ University of Kentucky, eseosa.ighodaro@uky.edu \\ Yasmin Hassoun \\ University of Kentucky \\ Danah Aldeiri \\ University of Kentucky \\ Janna H. Neltner \\ University of Kentucky, jmhack0@pop.uky.edu
}

See next page for additional authors

Follow this and additional works at: https://uknowledge.uky.edu/scobirc_facpub

Part of the Neuroscience and Neurobiology Commons

Right click to open a feedback form in a new tab to let us know how this document benefits you.

\section{Repository Citation}

Bachstetter, Adam D.; Ighodaro, Eseosa T.; Hassoun, Yasmin; Aldeiri, Danah; Neltner, Janna H.; Patel, Ela; Abner, Erin L.; and Nelson, Peter T., "Rod-Shaped Microglia Morphology Is Associated with Aging in 2 Human Autopsy Series" (2017). Spinal Cord and Brain Injury Research Center Faculty Publications. 28. https://uknowledge.uky.edu/scobirc_facpub/28

This Article is brought to you for free and open access by the Spinal Cord and Brain Injury Research at UKnowledge. It has been accepted for inclusion in Spinal Cord and Brain Injury Research Center Faculty Publications by an authorized administrator of UKnowledge. For more information, please contact UKnowledge@lsv.uky.edu. 
Rod-Shaped Microglia Morphology Is Associated with Aging in 2 Human Autopsy Series

\section{Digital Object Identifier (DOI)}

https://doi.org/10.1016/j.neurobiolaging.2016.12.028

Notes/Citation Information

Published in Neurobiology of Aging, v. 52, p. 98-105.

(c) 2017 Elsevier Inc. All rights reserved.

This manuscript version is made available under the CC-BY-NC-ND 4.0 license https://creativecommons.org/licenses/by-nc-nd/4.0/.

The document available for download is the author's post-peer-review final draft of the article.

Authors

Adam D. Bachstetter, Eseosa T. Ighodaro, Yasmin Hassoun, Danah Aldeiri, Janna H. Neltner, Ela Patel, Erin L. Abner, and Peter T. Nelson 
Published in final edited form as:

Neurobiol Aging. 2017 April ; 52: 98-105. doi:10.1016/j.neurobiolaging.2016.12.028.

\title{
Rod-shaped microglia morphology is associated with aging in two human autopsy series
}

\author{
Adam D. Bachstetter ${ }^{1,2,{ }^{*}, \text { Eseosa T. Ighodaro }}{ }^{1,3}$, Yasmin Hassoun ${ }^{1}$, Danah Aldeiri ${ }^{1}$, Janna \\ H. Neltner ${ }^{3,4}$, Ela Patel ${ }^{3}$, Erin L. Abner ${ }^{3,5}$, and Peter T. Nelson ${ }^{3,4}$ \\ ${ }^{1}$ Spinal cord and brain injury research center, University of Kentucky, Lexington, KY, USA \\ ${ }^{2}$ Department of Neuroscience, University of Kentucky, Lexington, KY, USA \\ ${ }^{3}$ Sanders-Brown Center on Aging, University of Kentucky, Lexington, KY, USA \\ ${ }^{4}$ Department of Pathology and Laboratory Medicine, Division of Neuropathology, University of \\ Kentucky, Lexington, KY, USA \\ ${ }^{5}$ Department of Epidemiology, University of Kentucky, Lexington, KY, USA
}

\begin{abstract}
A subtype of microglia is defined by the morphological appearance of the cells as rod-shaped. Little is known about this intriguing cell type, as there are only a few case reports describing rodshaped microglia in the neuropathological literature. Rod-shaped microglia were shown recently to account for a substantial proportion of the microglia cells in the hippocampus of both demented and cognitively intact aged individuals. We hypothesized that aging could be a defining feature in the occurrence of rod-shaped microglia. To test this hypothesis, two independent series of autopsy cases (total $n=168$ cases), which covered the adult lifespan from $20-100+$ years old, were included in the study. The presence or absence of rod-shaped microglia was scored on IBA1 immunohistochemically stained slides for the hippocampus and cortex. We found that age was one of the strongest determinants for the presence of rod-shaped microglia in the hippocampus and the cortex. We found no association with the presence of rod-shaped microglia and a self-reported history of a TBI. Alzheimer's disease related pathology was found to influence the presence of rod-shaped microglia, but only in the parietal cortex and not in the hippocampus or temporal cortex. Future studies are warranted to determine the functional relevance of rod-shaped microglia in supporting the health of neurons in the aged brain, and the signaling processes that regulate the formation of rod-shaped microglia.
\end{abstract}

\footnotetext{
*Correspondence: Adam D. Bachstetter, University of Kentucky, Spinal cord and brain injury research center, and Department of Neuroscience, B459 BBSRB, 741 S. Limestone St., Lexington, KY, 40536 USA, adam.bachstetter@uky.edu.

Conflict of Interest: None

We confirm that data contained in the manuscript being submitted have not been previously published, have not been submitted elsewhere and will not be submitted elsewhere while under consideration at Neurobiology of Aging.

All authors have reviewed the manuscript and approve of its contents and validate the accuracy of the data.

Publisher's Disclaimer: This is a PDF file of an unedited manuscript that has been accepted for publication. As a service to our customers we are providing this early version of the manuscript. The manuscript will undergo copyediting, typesetting, and review of the resulting proof before it is published in its final citable form. Please note that during the production process errors may be discovered which could affect the content, and all legal disclaimers that apply to the journal pertain.
} 


\section{Keywords}

Aging; microglia activation; neurodegeneration; neuroinflammation; neuropathology; hippocampus; Alzheimer's disease; traumatic brain injury

\section{Introduction}

Microglia are the resident tissue macrophage of the central nervous system (CNS). In the healthy CNS, microglia form a network of nearly uniformly distributed cells throughout the tissue, with thin highly ramified cell processes. Changes in microglia morphology away from the ramified or 'surveying' type of cell are well described in the literature, but largely center around the hypertrophic or 'activated' morphology. Despite recent studies defining a number of additional microglia morphologies (Bachstetter et al., 2015, Roth et al., 2014, Streit, 2006, Ziebell et al., 2012), little is known about the relevance of these morphological changes to human brain health and disease.

First described by Franz Nissl over 100 years ago (reviewed in (Graeber, 2010)), rod-shaped microglia are a particularly intriguing morphologically-defined subtype. The modern literature describing rod-shaped microglia is sparse, and is dominated by case reports, with the exception of a recent study that determined the relative amount of rod-shaped microglia in the hippocampus of different age-related neurodegenerative diseases (Bachstetter et al., 2015). Rod-shaped microglia were found in approximately $60 \%$ of the cases, including a subset of non-demented control cases, as well as in cases with different neurodegenerative disease (Bachstetter et al., 2015). The high prevalence of rod-shaped microglia in individuals 65 years or older suggested that aging or an age-related degenerative process might be an important predictor for the presence of rod-shaped microglia.

In this study we sought to determine if aging, Alzheimer's disease, or traumatic brain injury (TBI) could be a defining feature in the occurrence of rod-shaped microglia in the human brain. To this end we used two independent series of cases. The first series included 61 cases that covered the adult lifespan from 20 - 96 years of age, which were free of advanced neurodegenerative pathology. The second set of 107 cases were from an aged populationbased series, with an age range of 77 - 100+ years old, which included non-demented controls and cases with Alzheimer's disease. We found that older chronological age was a strong predictor for the presence of rod-shaped microglia, even when controlling for Alzheimer's disease pathology. Our data suggest that there may be an age-related change to neurons or microglia, which we have yet to define, that predisposes the aged brain to the presence of rod shaped microglia.

\section{Materials and Methods}

\subsection{UK series: University of Kentucky human subjects, and tissue processing}

A set of 61 autopsy cases were collected from the University of Kentucky (UK) bio tissue repository (Table 1). The cases were selected to cover the adult lifespan from 20 - 96 years of age. Cases were selected by the investigators (JHN and PTN) to be free of advanced neurodegenerative pathology. Exclusion criteria included pathologically confirmed 
neurodegenerative disease: specifically, but not limited to, advance disease pathology associated with Alzheimer's disease, dementia with Lewy bodies, hippocampal sclerosis of aging, and vascular dementia. To identify rod-shaped microglia, brains were stained with the IBA1 (ionized calcium binding adaptor molecule 1) antibody, which is used as a pan marker of macrophages / microglia in the brain. Paraffin-embedded tissue was processed, $8 \mu \mathrm{m}$-thick sections were cut, and immunohistochemical (IHC) staining was done using the primary antibody: IBA1 (rabbit polyclonal, 1:1,000 IHC, Wako Pure Chemical Industries, Richmond, VA). A biotinylated secondary antibody (Vector Laboratories) was amplified using avidinbiotin substrate (ABC solution, Vector Laboratories catalog no. PK-6100), followed by color development in Nova Red (Vector Laboratories). The Aperio ScanScope XT digital slidescanner was used to image the entire stained slide at 40x magnification to create a single high-resolution digital image. A tissue section from the hippocampus and the frontal cortex was analyzed by two observers blind to the experimental conditions (YH and DA). The observers exhaustively inspected the gray matter of the entire slides for the presence of rod-shaped microglia at a minimum of $8 \mathrm{x}$ magnification. Presence of rod-shaped microglia was defined as at least 1 clearly defined rod-shaped microglia: Figure 1 shows examples of cells defined as rod-shaped microglia.

\subsection{Al-ACT series: Aging, Dementia and Traumatic Brain Injury study of the Allen Institute (Al) for Brain Science}

The Aging, Dementia and Traumatic Brain Injury Study is a series of 107 autopsy cases with and without a history of traumatic brain injury drawn from the Adult Changes in Thought (ACT) study (AI-ACT)) (Table 1). Data used for the current project are publically available through the AI website (@ 2016 Allen Institute for Brain Science. Aging, Dementia and TBI. Available from: http://aging.brain-map.org/overview/explore). High magnification digital histopathological images of the entire physical slides are available to download for three brain regions-hippocampus, temporal cortex, and parietal cortex-for a number of neuropathological stains. Additional information is available in the AI-ACT database, including results from extensive biochemical assays, and clinical information about the cases. To determine how often rod-shaped microglia were found in this sample population, the digital histopathological images of IBA1 for the hippocampus, temporal cortex and parietal cortex were downloaded (all regions were not available for all cases), and were independently scored by three observers blind to the experimental conditions (YH, DA, and ADB) for the presence or absence of rod-shaped microglia in the gray matter. Presence of rod-shaped microglia was defined as at least 1 clearly defined rod-shaped microglia: Figure 1 shows examples of cells defined as rod-shaped microglia.

\subsection{Statistics}

JMP Pro Software version 12.0 or SAS 9.4 (SAS Institute, Inc.; Cary, NC, USA) was used for statistical analysis. Statistical significance was set at 0.05 . Contingency tables of categorical variables (presence of rod shaped microglia, sex, age group, history of TBI) were compared using the Pearson chi-square test or Fisher's exact test. The Cochran-Armitage Test was used to assess for a linear trend in the association between age and frequency of rod-shaped microglia. Logistic regression was used to assess the variable of interest (age) and potential confounders (sex, dementia status, Braak neurofibrillary tangle (NFT) stage, 
and Consortium to Establish a Registry for Alzheimer's Disease (CERAD) neuritic plaque rating) and to estimate odds ratios (OR) for the presence of rod-shaped microglia. Age groups were generated by a median split into 20-69 years old and 70 years old or older for the UK series, and 70-89 years old and 90 years old or older group for the AI-ACT series. Availability of confounder variables differed in the UK and AI-ACT cohorts. For UK, only sex $($ coded male $=1$, female $=2$ ) was available for all cases. For the AI-ACT series, additional variables were available: sex was coded as male $=1$, female $=2$, Braak NFT stage was coded as low (stage 0/I/II), moderate (III/IV), or high (V/VI); CERAD neuritic plaque rating was coded as none, sparse, moderate, or frequent; dementia status was coded as no dementia vs. dementia (DSM-IV criteria). Multivariable regression models to adjust clinicopathological associations for potential confounding factors have been used multiple times by our group (Abner et al., 2016, Abner et al., 2014, Ighodaro et al., 2017, Nelson et al., 2010). Comparisons between the AI-ACT series versus UK series were done using a logistic regression to determine ORs for presence of rod-shaped microglia in the hippocampus of older aged individuals ( $70-89$ years old).

In preclinical animal models, TBI has been shown to be a strong initiator for the presence of rod-shaped microglia; therefore, we sought to determine if a history of TBI exposure in the AI-ACT series could be associated with the presence of rod-shaped microglia. As part of the ACT study design participants were asked at initial enrollment to the study and at the biannual re-evaluation about exposure to a brain injury that caused a loss of consciousness (Dams-O'Connor et al., 2013). Cases reporting a history of TBI with a loss of consciousness were compared to those without a history of TBI for the presence of rod-shaped microglia. To determine if more severe TBI could be associated with the presence of rod-shaped microglia, cases with a self-reported loss of consciousness of more than 10 minutes were compared to the cases with no TBI exposure.

\section{Results}

\subsection{Age is associated with increased presence of rod-shaped microglia in the University of Kentucky (UK) aging series}

The primary research question was to elucidate the clinical-pathological correlations associated with the occurrence of rod-shaped microglia in postmortem hippocampal and cortical tissue. We first sought to determine if aging was accompanied by an increase incidence of IBA1 ${ }^{+}$rod-shaped microglia. Examples of rod-shaped microglia identified in the tissue of the aging UK series are shown in Figure 1. In the hippocampus a significant trend for an increased occurrence of any rod-shaped microglia with increasing age was found (Fig 2A). Separating the cases into younger adults (20-69 years old; $n=35$ ) to the older adults (70+ years old; $n=26$ ), at the time of death (using a median split of the cases), we found a significantly greater proportion of any rod-shaped microglia in hippocampus of older adults $(46.2 \%)$ compared to younger adults (11.4\%) (Fig 2B). The age-related increase in the presence of rod-shape microglia remained significant when corrected for sex $(\mathrm{p}=0.005)$. The OR for presence of rod-shaped microglia in the older adults versus the younger adults was 6.64 (95\% confidence interval (CI) 1.94-27.25), and when corrected for sex the OR was 5.87 (95\% CI 1.66-24.8); the reduction in magnitude was likely due to the 
fact that females in the UK series were both more likely to be older and more likely to have rod-shaped microglia in the hippocampus. In the frontal cortex, a similar age-related increase in the proportion of individuals with any rod-shaped microglia was found (Fig 2CD); however, this increase was not statistically significant.

To further explore the age-related differences in the occurrence of rod-shaped microglia, the cases were subdivided by which brain region(s) rod-shaped microglia were found, as follows: 1) in the hippocampus only, 2) in the cortex only, or 3) in both the cortex and the hippocampus (Fig 3). In those individuals less than 70 years old, rod-shaped microglia were found in the frontal cortex, or the hippocampus, but not in both the hippocampus and frontal cortex (Fig 3). This was in contrast to the older adults, where 19.2\% of the cases were found to have rod-shaped microglia in the frontal cortex and hippocampus.

\subsection{Age, and not traumatic brain injury (TBI) or Alzheimer's disease related pathology, was associated with the presence of rod-shaped microglia in the Al-ACT series}

To further explore the occurrence of rod-shaped microglia in older individuals we used the public data repository released by the Allen Institute (AI) for Brain Science (@ 2016 Allen Institute for Brain Science. Aging, Dementia and TBI. Available from: http://aging.brainmap.org/overview/explore). Overall we found that the percentage of cases positive for rodshaped microglia in the hippocampus in the older aged individuals (70-89 years old) was higher in the AI-ACT series compared to the UK series (UK $=42.9 \% 9 / 21$; AI-ACT $=$ $65.1 \% 28 / 430$. The OR for AI-ACT ( $70-89$ years old) vs UK series ( $70-89$ years old) to have rod-shaped microglia in the hippocampus was 2.49 (95\% CI, 0.86-7.44). The data suggested that increased age and/or Alzheimer's disease pathology found in the AI-ACT series in comparison to the UK series (Table 1) may be associated with the increased presence of rod-shaped microglia in the AI-ACT series.

Since the AI-ACT series contained oldest-old individuals, we also sought to determine whether oldest-old age (90+ years old) was associated with increased presence of rodshaped microglia relative to younger old (70-89 years old). In the hippocampus, the unadjusted OR for $90+$ vs. 70-89 was 1.30 (95\% CI 0.54-1.86), suggesting no significant effect of oldest-old age on the presence of rod-shaped microglia in this region. In the parietal cortex, the OR was 0.86 (95\% CI 0.34-2.16), and in the temporal cortex the OR was 3.27 (95\% CI 1.43-7.73), which may suggest that age effects are region-specific (Table 2). To evaluate if age was independently associated with presence of rod-shaped microglia after controlling for Alzheimer's disease pathology in the AI-ACT series, a logistic regression analysis was done to determine if sex, Braak NFT Stage, CERAD neuritic plaque score, and dementia status were potential confounders of the age-microglia relationship. The age effect remained significant in the temporal cortex (Fig 4A, Table 2) after adjustment, but there were also strong effects for dementia and sparse and moderate plaques in the parietal cortex, although confidence intervals were wide (Table 2). There were no significant associations between rod-shaped microglia and AD pathology in the hippocampus or temporal cortex. In all three regions, men were less likely than women to have rod-shaped microglia, but this effect was only significant in the parietal cortex (Fig 4B, Table 2). 
Finally, comparing a self-reported history of TBI with a loss of consciousness to the presence of rod-shaped microglia yielded no significant association (Fig 5A). No association was found between the severity of TBI, as determined by an extended period of loss of consciousness and the presence of rod-shaped microglia (Fig 5B). As some of the cases with a history of TBI had the exposure more than 6 decades before their death, we sought to compare only those cases with a TBI more proximal to the time of death. However, no significant association was found between the presence of rod-shaped microglia, and the individuals who had a first TBI exposure after the age of 60 years old (Fig 5C).

\section{Discussion}

The present study investigated the clinical-pathological correlations associated with the occurrence of rod-shaped microglia in postmortem hippocampal and cortical tissue. Using two independent autopsy series, we found that age was one of the strongest determinants of the presence of rod-shaped microglia in the hippocampus and the cortex. We found no association with the presence of rod-shaped microglia and a self-reported history of a TBI. Alzheimer's disease related pathology was found to influence the presence of rod-shaped microglia, but only in the parietal cortex and not in the hippocampus or temporal cortex. This suggests that increased activation of microglia (number or hypertrophic morphology) as seen in Alzheimer's disease does not explain the increase number of rod-shaped microglia in the brain. This is in agreement with our previous study, where we found a very weak correlation between the total number of microglia in the CA1 region of the hippocampus and the number of rod-shaped microglia $\left(\mathrm{R}^{2}=0.008 ; \mathrm{p}=0.28\right.$ Spearman $\mathrm{r}$ ) (Bachstetter et al., 2015). Thus, our results highlight that rod-shaped microglia may be more common than once believed, particularly in individuals over age 70, and that the increased prevalence of rod-shaped microglia cannot be explained by simply larger numbers of microglia or more activated microglia.

\subsection{Potential functions of rod-shaped microglia}

Experimentally, the most faithful way to induce rod-shaped microglia is by an interruption in axonal transport either caused by a traumatic brain injury, optic nerve transection, or axotomy (Ackman et al., 2006, Bachstetter et al., 2015, de Hoz et al., 2013, Sanders et al., 2014, Streit et al., 1999, Yuan et al., 2015, Ziebell et al., 2012). Deficits in axonal transport are thought to be an early molecular mechanism leading to neuronal loss in age-related neurodegenerative diseases (Morfini et al., 2009). In the current study it is not possible to determine if the rod-shaped microglia are associated specifically with neurons with deficits in axonal transport. The occurrence of rod-shaped microglia in more than one brain region in older individuals, as we found in this study, could indicate an age-related brain-wide change inhibiting axonal transport. The lack of a strong association of rod-shaped microglia with any one neurodegenerative disease, as we found in this study and in our previous work (Bachstetter et al., 2015), could be explained by the shared degenerative mechanism of axonal transport deficits seen in aging and age-related neurodegenerative diseases (Morfini et al., 2009). 
The function of rod-shaped microglia is largely unknown. There has been some speculation that rod-shaped microglia might provide a protective function to neurons, potentially by surrounding less damaged neurons to promote survival of the neurons (Streit et al., 1999, Trapp et al., 2007, Ziebell et al., 2012). Mechanistically, the neuroprotection could be imparted through the process of synaptic stripping, to alter the excitability of the weakened neuron (Trapp et al., 2007). Rod-shaped microglia may also provide trophic support through some yet to be identified mechanism that would appear to be contact dependent (Streit et al., 1999). An intriguing possibility would be that rod-shaped microglia are involved in a process of mitochondria transfer, as was recently shown for astrocytes following stroke (Hayakawa et al., 2016), however more work is needed to test this possibility.

\subsection{Potential chemotaxis signals for the formation of rod-shaped microglia}

The presence of rod-shaped microglia associated with specific neurons, while adjacent neurons are not associated with rod-shaped microglia, would suggest an active neuronmicroglia signal regulating the specificity of rod-shaped microglia to individual neurons. Neurons are able to interact directly with microglia through a number of neuron-microglia specific signaling pathways. A well-documented example of such a signaling pathway is the neuron ligand, CX3CL1 and the microglia receptor, CX3CR1. The CX3CR1-CX3CL1 pathway is vital for the suppression of inflammatory responses from microglia. Importantly, this pathway has been shown to be dysfunctional with age (Bachstetter et al., 2011, Corona et al., 2010, Lyons et al., 2009). The age-related loss of CX3CL1 may reduce the contact inhibition signals, thereby priming the aged brain for the formation of rod-shaped microglia. Yet, rod-shaped microglia are not a defining feature in CX3CR1 KO mice (Cardona et al., 2006, Rogers et al., 2011), so it is unlikely that this pathway is a sole driver for the formation of rod-shaped microglia. Thus, there must also be a chemotaxis signal that is released from the neuron to cause the recruitment of the microglia to the neuron. As microglia, have been shown to constantly make brief contact with neurons (Tremblay et al., 2010), the chemotaxis signal could be contact dependent and not a soluble factor released from the neurons.

The AI-ACT study includes an extensive array of histological and biochemical information, including RNAseq. A superficial exploration of this data did not reveal any proteins or genes at the tissue level that were associated with the presence or absence of rod-shaped microglia. As rod-shaped microglia account for only a small percentage of the total microglia population (Bachstetter et al., 2015), and the total microglia population only accounts for 5$20 \%$ of the total glia population (van Rossum and Hanisch, 2004), it is likely at the tissue level a rod-shaped microglia molecular signature may not be evident. Future studies using laser capture microdissection would be needed to isolate individual neurons that are associated with rod-shaped microglia and adjacent neurons and microglia which are not in close association. Then a subtractive analysis could be done to determine the molecular signature unique to the neuron associated with the rod-shaped microglia and that of the rodshaped microglia, compared to the adjacent neurons and microglia. 


\subsection{Limitations and future directions}

A limitation of the study is that the AI-ACT series over-represents males and individuals with a history of TBI, compared to the community-based sample of the larger Adult Changes in Thought study. Methods for weighted analyses to extrapolate results to the larger cohort are provided by AI (@ 2016 Allen Institute for Brain Science. Aging, Dementia and TBI. Available from: http://aging.brain-map.org/overview/explore), but were not used for this study. Therefore, findings reported should be seen as specific to the AI-ACT series and may not be representative of the larger cohort or the population in general. A second limitation of our study is that more than half of the cases with a history of TBI, had the first exposure to TBI many decades before death; thus, it is reasonable to assume that rod-shaped microglia may have resolved over time. A third limitation of our study was that it was not possible with the tissue available to provide a design-based stereological quantification of the total number of microglia; thus, we cannot account for the effect of age-related increase in microglia density that may influence the likelihood of detecting rod-shaped microglia in the older aged individuals.

A striking finding was the lack of rod-shaped microglia in the hippocampus of individuals aged 40-69 years old. Leading causes of death are known to change across age groups. Therefore, we hypothesized that differences in cause of death in the 40-69 years old group could be associated with the lack of rod-shaped microglia in this group. In the younger individuals (aged 20-69 years old), rod-shaped microglia were not found in the cases where the cause of death was either cardiac associated, drug abuse, or sepsis. Rod-shaped microglia were found in cases of pulmonary embolism, liver failure, or cancer. A limitation of this study was that cause of death was known for only a subset of the individuals 60 years old or older, so associations between cause of death and rod-shaped microglia could not be fully determined. Future studies specifically investigating the relationship of cause of death and rod-shaped microglia may be warranted.

\subsection{Conclusion}

In summation, our results highlight that aging, but not necessarily Alzheimer's disease or a history of TBI, is a defining feature in the presence of rod-shaped microglia in the human brain. Future studies are warranted to determine the functional relevance of rod-shaped microglia in supporting the health of neurons in the aged brain, and the signaling processes that regulate the formation of rod-shaped microglia.

\section{Acknowledgments}

We are profoundly grateful to all of the study participants who make this research possible. The corresponding author, Adam Bachstetter, $\mathrm{PhD}$, had full access to all of the data in the study and takes responsibility for the integrity of the data and the accuracy of the data analysis. Research reported in this publication was supported by National Institutes of Health under award numbers P30 AG028383, R00 AG044445. The content is solely the responsibility of the authors and does not represent the official views of the National Institutes of Health.

\section{References}

Abner EL, Nelson PT, Kryscio RJ, Schmitt FA, Fardo DW, Woltjer RL, Cairns NJ, Yu L, Dodge HH, Xiong C, Masaki K, Tyas SL, Bennett DA, Schneider JA, Arvanitakis Z. Diabetes is associated with 
cerebrovascular but not Alzheimer's disease neuropathology. Alzheimers Dement. 2016; 12(8):8829. [PubMed: 26812281]

Abner EL, Nelson PT, Schmitt FA, Browning SR, Fardo DW, Wan L, Jicha GA, Cooper GE, Smith CD, Caban-Holt AM, Van Eldik LJ, Kryscio RJ. Self-reported head injury and risk of late-life impairment and AD pathology in an AD center cohort. Dement Geriatr Cogn Disord. 2014; 37(56):294-306. [PubMed: 24401791]

Ackman JB, Siddiqi F, Walikonis RS, LoTurco JJ. Fusion of microglia with pyramidal neurons after retroviral infection. J Neurosci. 2006; 26(44):11413-22. [PubMed: 17079670]

Bachstetter AD, Morganti JM, Jernberg J, Schlunk A, Mitchell SH, Brewster KW, Hudson CE, Cole MJ, Harrison JK, Bickford PC, Gemma C. Fractalkine and CX3CR1 regulate hippocampal neurogenesis in adult and aged rats. Neurobiol Aging. 2011; 32(11):2030-44. [PubMed: 20018408]

Bachstetter AD, Van Eldik LJ, Schmitt FA, Neltner JH, Ighodaro ET, Webster SJ, Patel E, Abner EL, Kryscio RJ, Nelson PT. Disease-related microglia heterogeneity in the hippocampus of Alzheimer's disease, dementia with Lewy bodies, and hippocampal sclerosis of aging. Acta Neuropathol Commun. 2015:3. [PubMed: 25627160]

Cardona AE, Pioro EP, Sasse ME, Kostenko V, Cardona SM, Dijkstra IM, Huang D, Kidd G, Dombrowski S, Dutta R, Lee JC, Cook DN, Jung S, Lira SA, Littman DR, Ransohoff RM. Control of microglial neurotoxicity by the fractalkine receptor. Nat Neurosci. 2006; 9(7):917-24. [PubMed: 16732273]

Corona AW, Huang Y, O’Connor JC, Dantzer R, Kelley KW, Popovich PG, Godbout JP. Fractalkine receptor (CX3CR1) deficiency sensitizes mice to the behavioral changes induced by lipopolysaccharide. J Neuroinflammation. 2010; 7:93. [PubMed: 21167054]

Dams-O'Connor K, Gibbons LE, Bowen JD, McCurry SM, Larson EB, Crane PK. Risk for late-life reinjury, dementia and death among individuals with traumatic brain injury: a population-based study. J Neurol Neurosurg Psychiatry. 2013; 84(2):177-82. [PubMed: 23172868]

de Hoz R, Gallego BI, Ramirez AI, Rojas B, Salazar JJ, Valiente-Soriano FJ, Aviles-Trigueros M, Villegas-Perez MP, Vidal-Sanz M, Trivino A, Ramirez JM. Rod-Like Microglia Are Restricted to Eyes with Laser-Induced Ocular Hypertension but Absent from the Microglial Changes in the Contralateral Untreated Eye. Plos One. 2013; 8(12)

Graeber MB. Changing Face of Microglia. Science. 2010; 330(6005):783-8. [PubMed: 21051630]

Hayakawa K, Esposito E, Wang X, Terasaki Y, Liu Y, Xing C, Ji X, Lo EH. Transfer of mitochondria from astrocytes to neurons after stroke. Nature. 2016; 535(7613):551-5. [PubMed: 27466127]

Ighodaro ET, Abner EL, Fardo DW, Lin AL, Katsumata Y, Schmitt FA, Kryscio RJ, Jicha GA, Neltner JH, Monsell SE, Kukull WA, Moser DK, Appiah F, Bachstetter AD, Van Eldik LJ, Nelson PT. Alzheimer's Disease Neuroimaging I. Risk factors and global cognitive status related to brain arteriolosclerosis in elderly individuals. J Cereb Blood Flow Metab. 2017; 37(1):201-16. [PubMed: 26738751]

Lyons A, Lynch AM, Downer EJ, Hanley R, O’Sullivan JB, Smith A, Lynch MA. Fractalkine-induced activation of the phosphatidylinositol-3 kinase pathway attentuates microglial activation in vivo and in vitro. J Neurochem. 2009; 110(5):1547-56. [PubMed: 19627440]

Morfini GA, Burns M, Binder LI, Kanaan NM, LaPointe N, Bosco DA, Brown RH Jr, Brown H, Tiwari A, Hayward L, Edgar J, Nave KA, Garberrn J, Atagi Y, Song Y, Pigino G, Brady ST. Axonal transport defects in neurodegenerative diseases. J Neurosci. 2009; 29(41):12776-86. [PubMed: 19828789]

Nelson PT, Schmitt FA, Jicha GA, Kryscio RJ, Abner EL, Smith CD, Van Eldik LJ, Markesbery WR. Association between male gender and cortical Lewy body pathology in large autopsy series. J Neurol. 2010; 257(11):1875-81. [PubMed: 20563821]

Rogers JT, Morganti JM, Bachstetter AD, Hudson CE, Peters MM, Grimmig BA, Weeber EJ, Bickford PC, Gemma C. CX3CR1 deficiency leads to impairment of hippocampal cognitive function and synaptic plasticity. J Neurosci. 2011; 31(45):16241-50. [PubMed: 22072675]

Roth TL, Nayak D, Atanasijevic T, Koretsky AP, Latour LL, McGavern DB. Transcranial amelioration of inflammation and cell death after brain injury. Nature. 2014; 505(7482):223-8. [PubMed: 24317693] 
Sanders DW, Kaufman SK, DeVos SL, Sharma AM, Mirbaha H, Li AM, Barker SJ, Foley AC, Thorpe JR, Serpell LC, Miller TM, Grinberg LT, Seeley WW, Diamond MI. Distinct Tau Prion Strains Propagate in Cells and Mice and Define Different Tauopathies. Neuron. 2014; 82(6):1271-88. [PubMed: 24857020]

Streit WJ. Microglial senescence: does the brain's immune system have an expiration date? Trends Neurosci. 2006; 29(9):506-10. [PubMed: 16859761]

Streit WJ, Walter SA, Pennell NA. Reactive microgliosis. Prog Neurobiol. 1999; 57(6):563-81. [PubMed: 10221782]

Trapp BD, Wujek JR, Criste GA, Jalabi W, Yin XH, Kidd GJ, Stohlman S, Ransohoff R. Evidence for synaptic stripping by cortical microglia. Glia. 2007; 55(4):360-8. [PubMed: 17136771]

Tremblay ME, Lowery RL, Majewska AK. Microglial interactions with synapses are modulated by visual experience. PLoS Biol. 2010; 8(11):e1000527. [PubMed: 21072242]

van Rossum D, Hanisch UK. Microglia. Metab Brain Dis. 2004; 19(3-4):393-411. [PubMed: 15554430]

Yuan TF, Liang YX, Peng B, Lin B, So KF. Local proliferation is the main source of rod microglia after optic nerve transection. Sci Rep. 2015:5.

Ziebell JM, Taylor SE, Cao TX, Harrison JL, Lifshitz J. Rod microglia: elongation, alignment, and coupling to form trains across the somatosensory cortex after experimental diffuse brain injury. $\mathrm{J}$ Neuroinflammation. 2012:9. [PubMed: 22248083] 
- $\quad$ Rod-shaped microglia are associated with aging in human hippocampus and cortex.

- $\quad$ Alzheimer's disease influences the presence of rod-shaped microglia.

- A history of traumatic brain injury was not associated with rod-shaped microglia. 
A
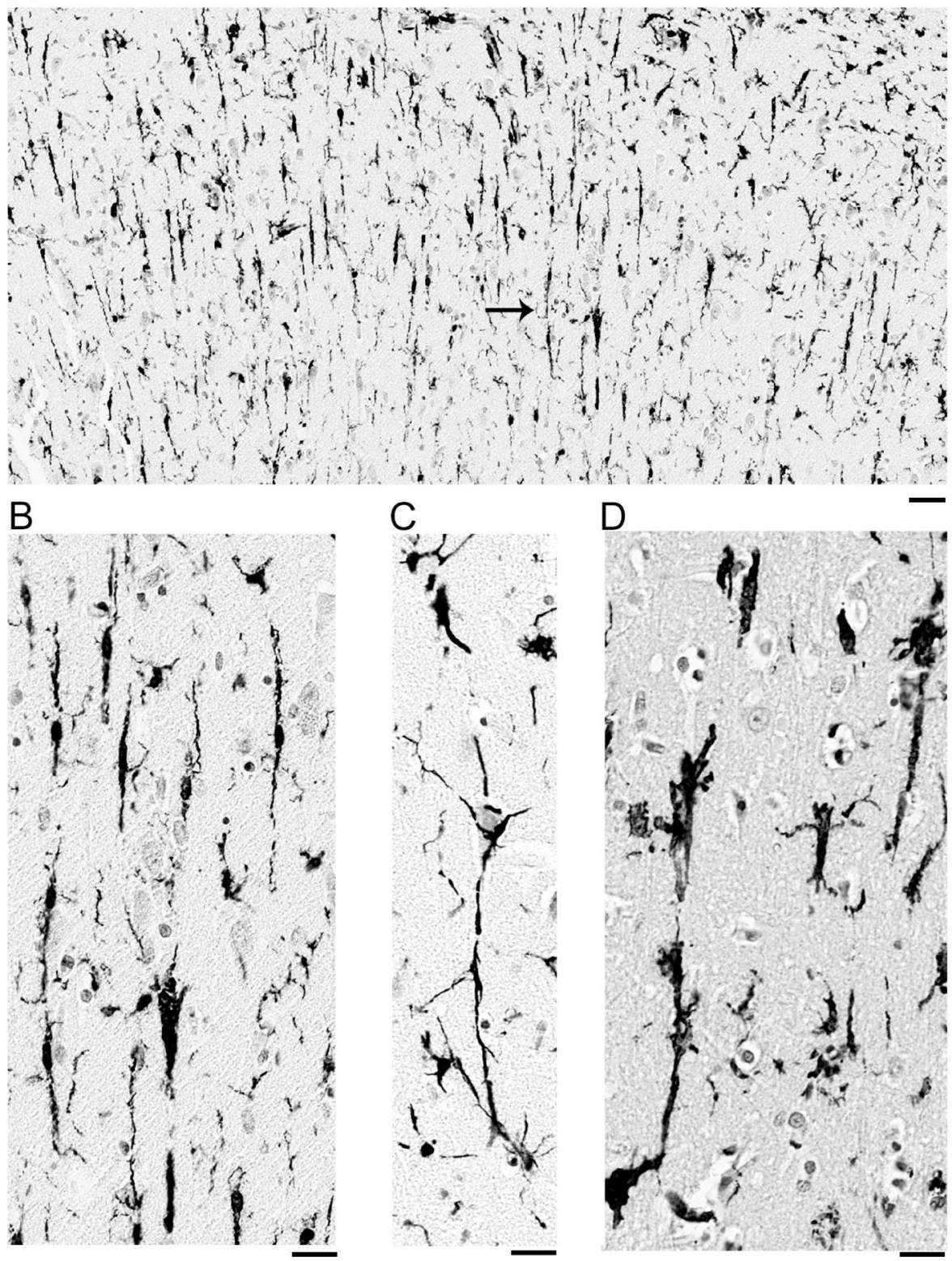

D

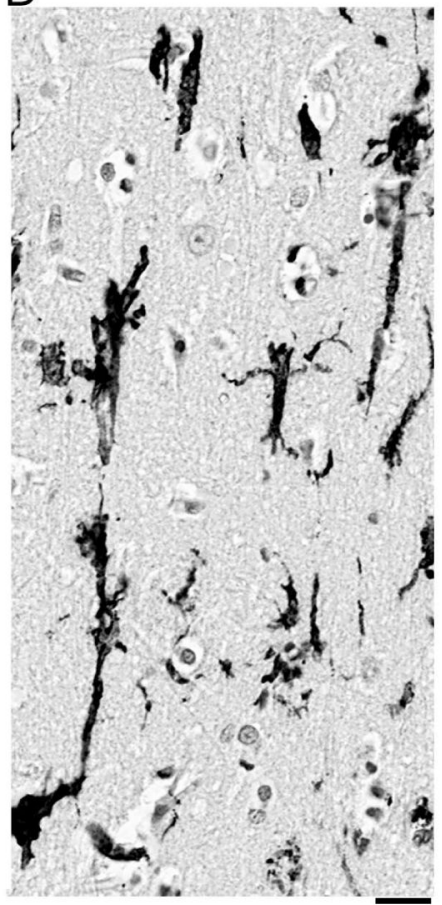

Figure 1. Examples of rod-shaped microglia from the UK series

(A) Numerous rod-shaped microglia are found throughout the CA1 region of the hippocampus of an 86-year-old female. The arrow indicates the area shown at a higher magnification in (B). (C) Long thin rod-shaped microglia, which appear to be surrounding a pyramidal neuron, in the subiculum of a 39-year-old male, who had hypoxia / ischemia seen in the frontal cortex of a 28-year-old female, who died of atherosclerotic disease. (E) A number of long trains of rod-shaped microglia were found in the CA1 region of the hippocampus of a 25-year-old female, who had hypoxia / ischemia pathology, and a pulmonary associated cause of death. Scale bar $=50 \mu \mathrm{m}$
E

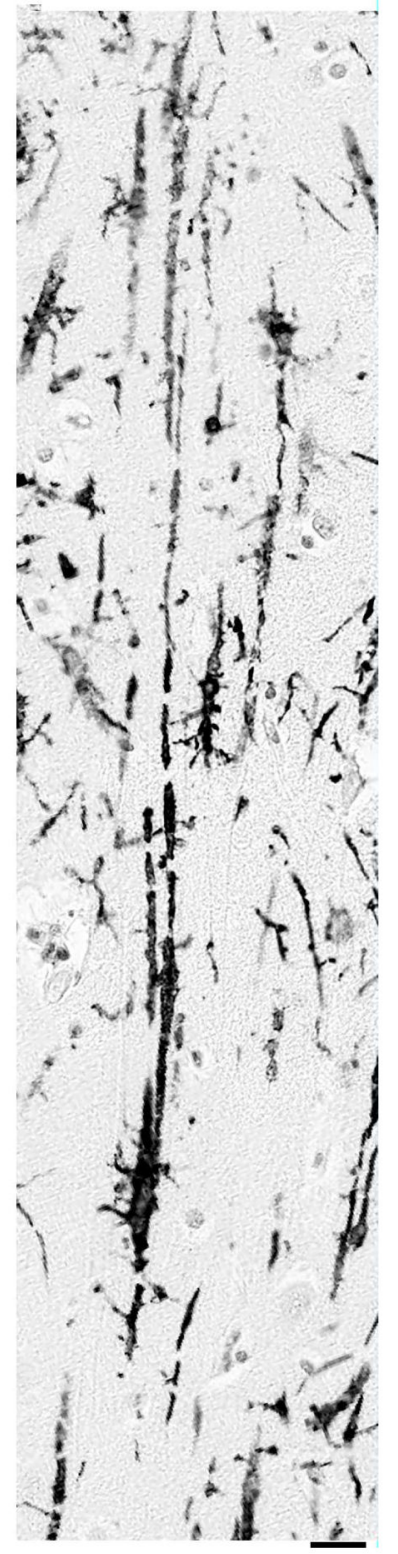
pathology, and a pulmonary associated cause of death. (D) Example of rod-shaped microglia 


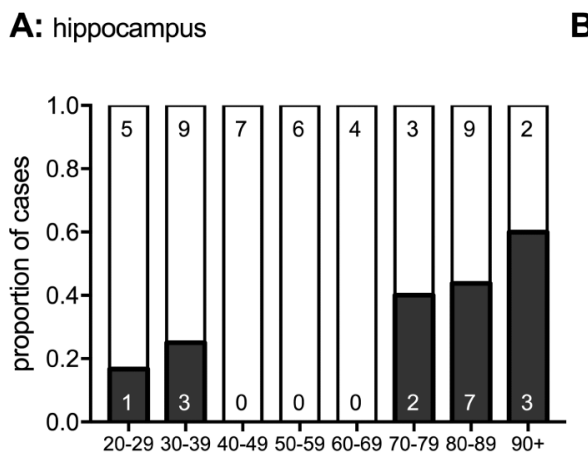

C: frontal cortex
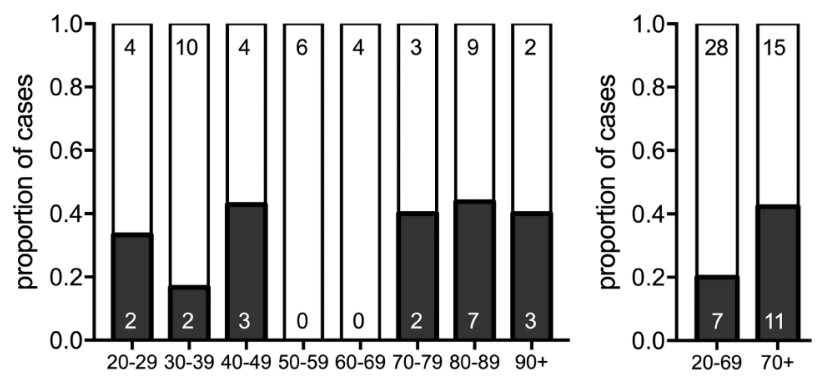

B: hippocampus

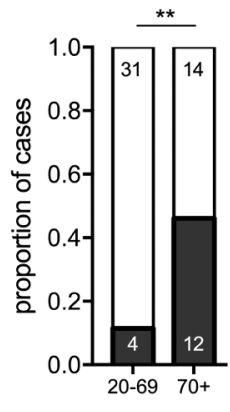

D: frontal cortex

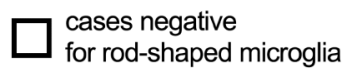

Figure 2. Association of aging on the presence of rod-shaped microglia

(A) In the hippocampus a significant trend was found for an increasing occurrence of rodshaped microglia with increasing age ( $\mathrm{p}=0.017$, Cochran Armitage Trend Test). (B) A significant increase in the occurrence of rod shaped microglia in the hippocampus was found in those individuals 70-years or older (70+) at the time of death, compared to those individuals 20-69-years old at the time of death ( $\mathrm{p}=0.0033$, Fisher Exact test) (C) A similar pattern was found for the frontal cortex when plotted as age by decade ( $p=0.222$, CochranArmitage Trend Test), or split into young adults (20-69-years old) and older adults (70+) $(\mathrm{p}=0.089$, Fisher Exact test). The number of cases in each group are shown in the bars. 


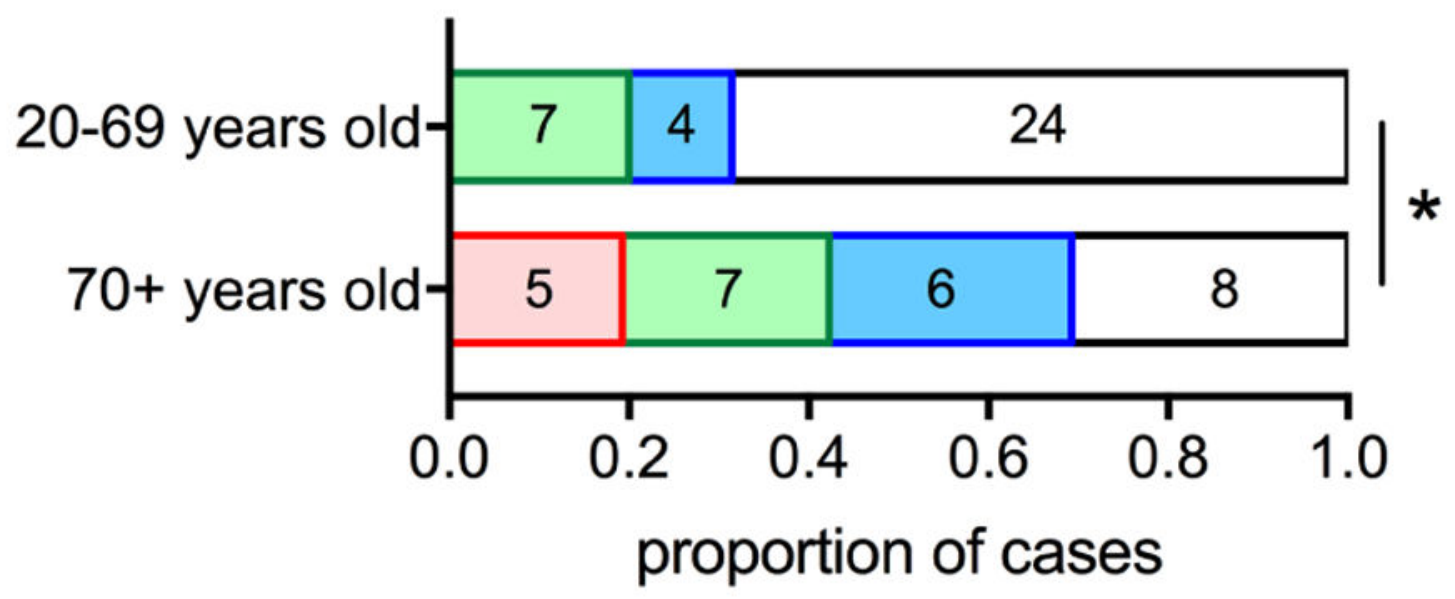

$\square$ negative for rod-shaped microglia

positive for rod-shaped microglia in hippocampus only

positive for rod shaped microglia in cortex only

$\square$ positive for rod shaped microglia in cortex and hippocampus

Figure 3. Regional heterogeneity in the occurrence of rod-shaped microglia in younger adults versus aged adults

Cases were subdivided by which brain region(s) rod-shaped microglia were found, as

follows: 1) in both the cortex and the hippocampus, 2) in the hippocampus only, 3) in the cortex only. In younger adults (20-69 years old) rod shaped microglia were found in the hippocampus, or in the frontal cortex, which is in contrast to the older adults (70+ years old) $\left(p=0.047, \chi^{2}\right.$ test). The number of cases in each group are shown in the bars. 


\section{A: age}

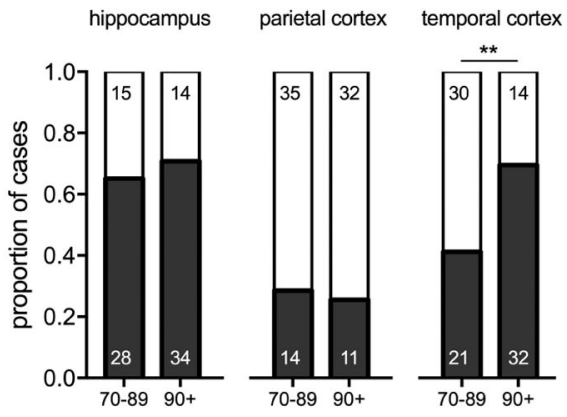

B: $\operatorname{sex}$

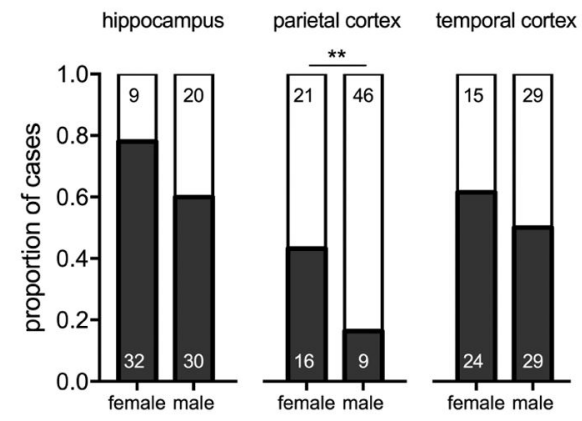

cases negative for rod-shaped microglia in hippocampus or cortex

cases positive for rod-shaped microglia in hippocampus or cortex

Figure 4. Association of age and sex on the presence of rod-shaped microglia The presence of rod-shaped microglia was determined in cases from the AI-ACT series. (A) Age at the time of death was found to significantly contribute to an increase percentage of cases positive for the presence of rod-shaped microglia. (B) Sex was also found to effect the odds ratio that rod-shaped microglia would be found. See table 2 for summary or results. The number of cases in each group are shown in the bars. 
A: TBI with loss of consciousness

hippocampus parietal cortex temporal cortex

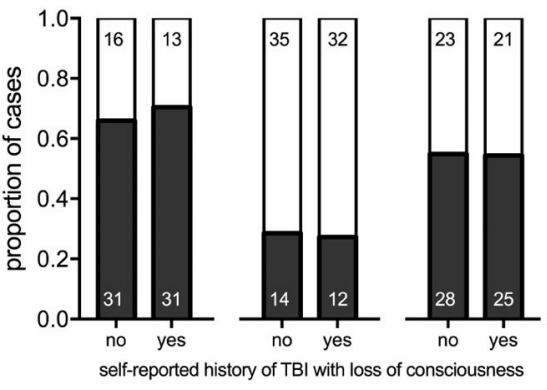

B: $\mathrm{TBI}$ with loss of consciousness $>10 \mathrm{~min}$

hippocampus parietal cortex temporal cortex

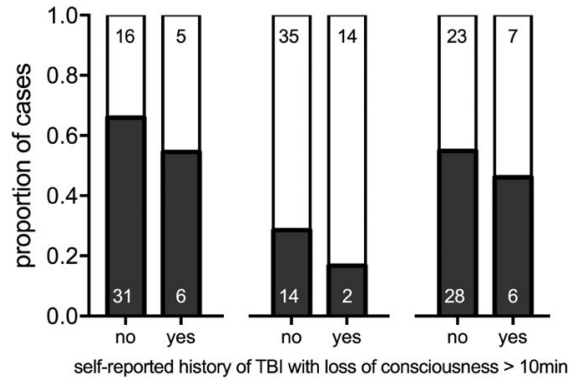

C: TBI with loss of consciousness after the age of 60 years old

hippocampus parietal cortex temporal cortex

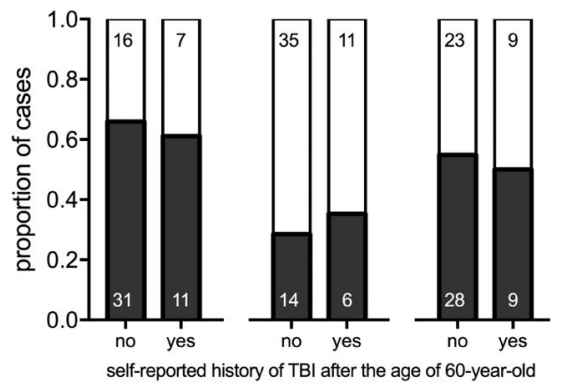

cases negative

for rod-shaped microglia

in hippocampus or cortex

cases positive

for rod-shaped microglia

in hippocampus or cortex

Figure 5. Association between a history of traumatic brain injury (TBI) and the presence of rodshaped microglia in AI-ACT series

(A) A self-reported history of at least one TBI with a loss of consciousness of at least a few seconds was not found associated with the presence of rod-shaped microglia. No association with the presence of rod-shaped microglia was found with either: (B) a self-reported history of at least one TBI with a loss of consciousness of more than $10 \mathrm{~min}$; or a first TBI exposure after the age of 60 years old. The number of cases in each group are shown in the bars. 


\section{Table 1}

Case series characteristics

\begin{tabular}{|c|c|c|}
\hline & UK series & AI-ACT series \\
\hline Number of cases & 61 & 107 \\
\hline \multicolumn{3}{|l|}{ Age } \\
\hline $20-29$ & $6(9.8 \%)$ & $0(0 \%)$ \\
\hline 30-39 & $12(19.7 \%)$ & $0(0 \%)$ \\
\hline $40-49$ & $7(11.5 \%)$ & $0(0 \%)$ \\
\hline $50-59$ & $6(9.8 \%)$ & $0(0 \%)$ \\
\hline $60-69$ & $4(6.6 \%)$ & $0(0 \%)$ \\
\hline 70-79 & $5(8.2 \%)$ & $12(11.2 \%)$ \\
\hline $80-89$ & $16(26.2 \%)$ & $45(42.1 \%)$ \\
\hline 90-99 & $5(8.2 \%)$ & $43(40.2 \%)$ \\
\hline $100+$ & $0(0 \%)$ & $7(7.5 \%)$ \\
\hline \multicolumn{3}{|l|}{ Sex } \\
\hline Male & $27(43.3 \%)$ & $63(58.9 \%)$ \\
\hline Female & $31(50.8 \%)$ & $44(41.1 \%)$ \\
\hline \multicolumn{3}{|l|}{ Dementia status } \\
\hline No dementia & $61(100 \%)$ & $57(53.3 \%)$ \\
\hline Dementia & $0(0 \%)$ & $50(46.7 \%)$ \\
\hline \multicolumn{3}{|l|}{ Braak NFT stage } \\
\hline 0/I/II & $61(100 \%)$ & $28(26.2 \%)$ \\
\hline III/IV & $0(0 \%)$ & $44(41.1 \%)$ \\
\hline V/VI & $0(0 \%)$ & $32(29.9 \%)$ \\
\hline \multicolumn{3}{|l|}{ CERAD rating } \\
\hline None & $61(100 \%)$ & $24(22.4 \%)$ \\
\hline Sparse & $0(0 \%)$ & $33(30.8 \%)$ \\
\hline Moderate & $0(0 \%)$ & $25(23.4 \%)$ \\
\hline Frequent & $0(0 \%)$ & 25 (23.4\%) \\
\hline
\end{tabular}

Number of cases and percent of total cases for the series are shown in the table. Abbreviations: University of Kentucky bio tissue repository series of cases (UK series). The Aging, Dementia and Traumatic Brain Injury Study series (๑ 2016 Allen Institute for Brain Science. Aging, Dementia and TBI. Available from: http://aging.brain-map.org/overview/explore) (AI-ACT series). Braak neurofibrillary tangle (NFT) Stage. Consortium to Establish a Registry for Alzheimer's Disease (CERAD) 

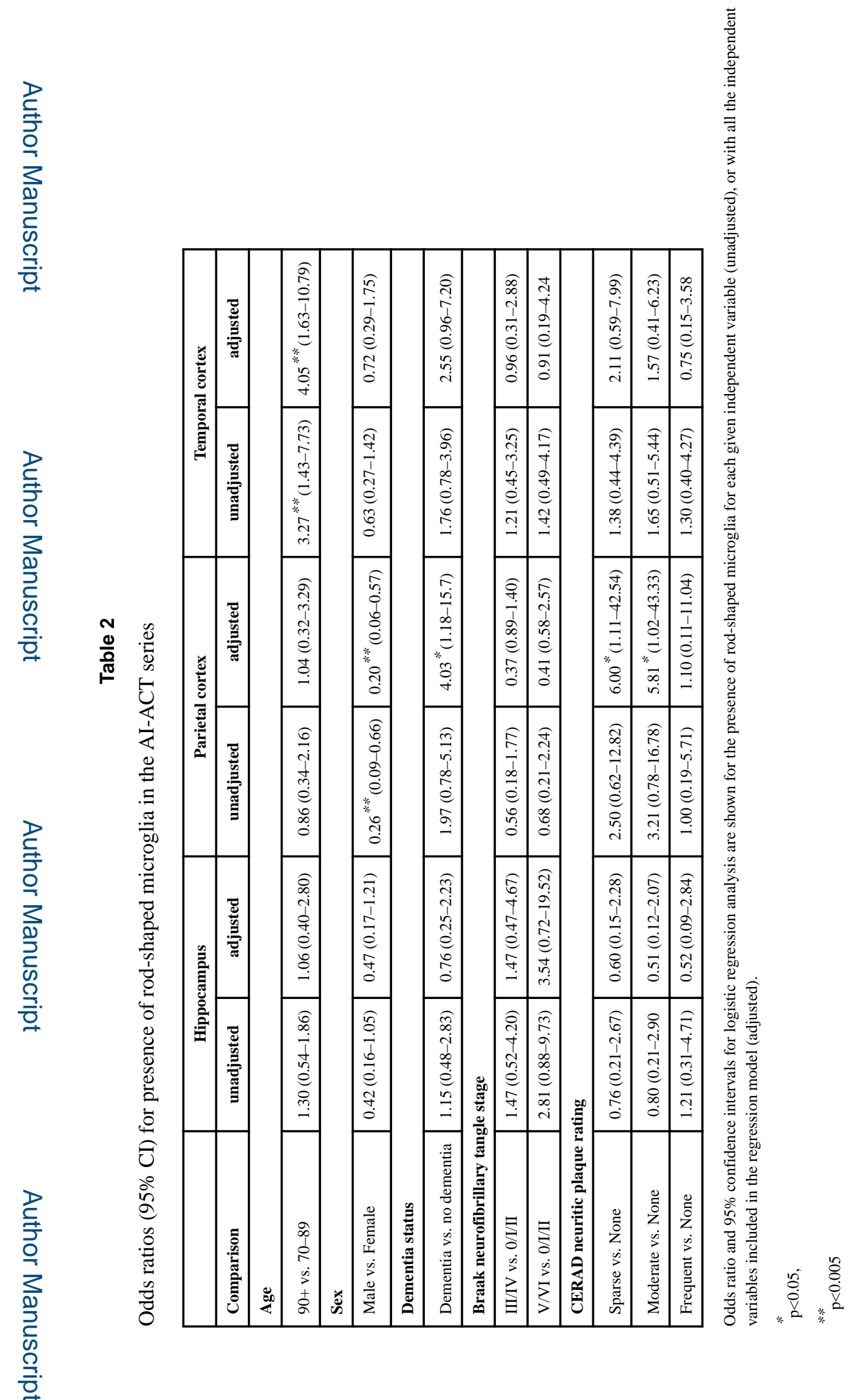

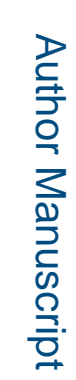

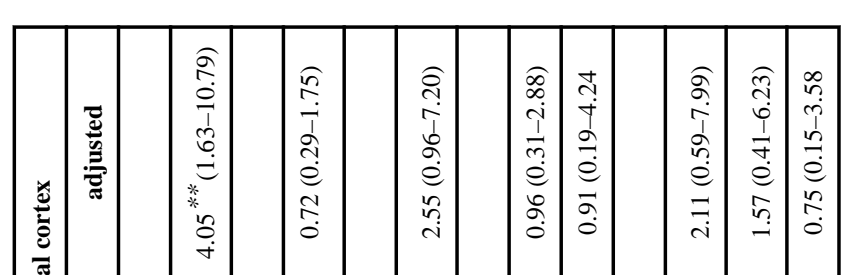

ב্

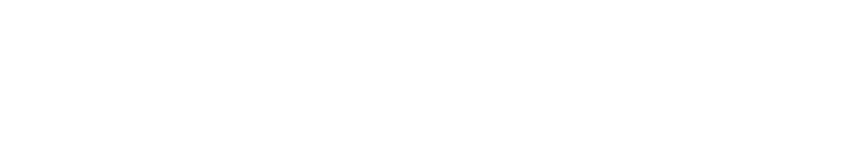

Neurobiol Aging. Author manuscript; available in PMC 2018 April 01. 